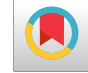

\title{
Assessing the Online Outpatient Booking System
}

\author{
Mahnaz Samadbeik, ${ }^{1}$ Marzieh Saremian, ${ }^{2}$ Ali Garavand, ${ }^{3}$ Negar Hasanvandi, ${ }^{4}$ Sahar Sanaeinasab, ${ }^{4}$ and \\ Hadis Tahmasebi ${ }^{4, *}$ \\ ${ }^{1}$ Social Determinants of Health Research Center, Lorestan University of Medical Sciences, Khorramabad, Iran \\ ${ }^{2}$ Asadabad School of Medical Sciences, Asadabad, Iran \\ ${ }^{3}$ Department of Health Information Management and Technology, School of Allied Medical Sciences, Shahid Beheshti University of Medical Sciences, Tehran, Iran \\ ${ }^{4}$ Student Research Committee, Lorestan University of Medical Sciences, Khorramabad, Iran \\ "Corresponding author: Hadis Tahmasebi, Student Research Committee, Lorestan University of Medical Sciences, Khorramabad, Iran. E-mail: virya67@gmail.com
}

Received 2017 August 14; Revised 2017 November 26; Accepted 2017 December 23.

\begin{abstract}
Background: Appointment scheduling is considered as one of the important aspects of patient flow management. Today, the advent of Internet into the realm of health care has paved the way for employing an internet-based booking system for visiting physicians. Therefore, this study aimed at assessing the online outpatient booking system in Iranian hospitals.

Methods: The present study was an applied descriptive study. In this study, all outpatient appointment booking websites in Iranian hospitals were evaluated. The methods of outpatients booking in the hospitals $(\mathrm{N}=598)$ were determined based on information available at their active websites. Finally, 31 active online booking systems were identified and investigated using a localized checklist, including 59 items in 6 sections.

Results: The results showed that only $13.03 \%$ of Iranian hospitals had an active online booking system. All the online booking systems had a weak quality based on the total score percentage (17.10\%). The highest score percentages were related to sections of online security features (45\%) and details of health insurance (42\%), while the clinical data section received the lowest score (7.25\%). The most frequent item was the patient's name in the patient demographics section (27 websites).

Conclusions: The outpatient appointment system in Iranian hospitals is done more traditionally through walk-ins to the health centers. The online outpatient booking systems have poor quality. Hence, codification and communication of national standards for designing hospital websites, periodical evaluation of appointment booking websites by ministry of health, and survey of the users of this method can enhance the qualitative level of these websites.
\end{abstract}

Keywords: Reservation, Electronic Appointment Booking, Outpatients

\section{Background}

Reservation means planning for the date, time and place of a clinical visit to receive healthcare services (1). In general, there are 2 types of medical appointments, including scheduled and unscheduled appointments. Making an unscheduled appointment needs no reservation and only requires the patient to visit the medical center while a scheduled appointment is done via the phone, SMS or the Internet (2). Since patient flow management requires attention to 3 important aspects of arrival of patients, service process, and queuing process in an outpatient unit, appointment scheduling is considered as one of the important aspects of patient workflow management, and a basic tool for controlling patient waiting times is in the process of receiving health care services. The waiting time to visit a physician is considered one of the most important indicators of patients' access to healthcare service (24). Also, simultaneous activities of several physicians in a hospital outpatient ward, necessitates the need to share resources, such as space, personnel and equipment, and setting appointments in the out-patient ward is of special importance 3.

Fax, phone and in person visits are among traditional appointment booking methods. These appointment booking systems are associated with many problems, such as long waiting times, poor quality of services, wasting time of the patient and physician, patient dissatisfaction, lack of integration of appointment booking system $(3,5,6)$, inefficient use of human resources, and inadequate management of health care institution (7). Today, computer programs and telecommunications are offered to improve the quality of health care services in developing countries (8). Online appointment booking systems, because of their flexibility in planning and time efficienct (9-13) are a successful solution for physicians and patients in setting clinical appointments (13). This system, has advantages and features, including access to the system at any 
time and any place, allowing the user to see the physicians' appointments (9), selection of the desired option, editing the recorded information, more favorable relationship between physician and patient, improvement of the efficiency and effectiveness of care (5), cost reduction (11$14)$, reduction of the number and volume of employees (7), saving patient information for use in subsequent appointments (11), and reduction of the number of missed appointments $(9,11,13-18)$. However, despite the importance of this system, a few studies have been done on its evaluation and existing researches in this field have investigated topics, such as designing a web-based appointment system (19), patient satisfaction of online appointment booking systems $(4,20)$, development of information technology acceptance model for an online appointment booking system in hospitals (21-23), implementation and use of the online appointment booking system in clinics $(15,20)$, and improving the management of appointments $(24,25)$. There are limited studies in Iran on the need for smart systems to prepare an optimized program for patients and investigating the appointment-making system of private physicians' offices $(5,22)$. Increased satisfaction of the clients of healthcare centers is an important pillar of the "health transformation scheme" implemented by the ministry of health and Medical education. In this regard, all hospitals, with the aim of improving patient satisfaction, have been mandated by the ministry of health to convert their appointment system to an online system and this issue is considered in their validation. Therefore, given that setting up an online appointment booking system requires necessary infrastructure, the present research was designed to evaluate the online outpatient booking system in Iranian hospitals so that the results could be used to create an efficient and proper online appointment booking procedure and solve many appointment problems in outpatient wards.

\section{Methods}

The present study was an applied descriptive study, which was conducted in 2015. In this study, all outpatient appointment booking websites in Iranian hospitals were evaluated. To identify these websites, initially, a list of hospitals in the country and their characteristics were extracted from hospital statistics and information system (HSIS) (http://avab.behdasht.gov.ir), which included 1166 hospitals. These characteristics include the type of activity and dependency (collegiate-education, treatment, and non- collegiate), ownership type (affiliation to Medical Sciences, military, social, charity, private and other universities), type of activity (education-treatment and academictreatment), and type of care (general, specialized, and subspecialized). All extracted hospitals were evaluated in terms of having available the studied websites. This step was completed using the HSIS by entering the name of the hospital as a keyword in the Google search engine or referring to the content table of hospitals or health centers' website.

Since hospital websites should provide the most updated and the most accurate information 24, outpatient appointment procedures in hospitals with an active website was designated based on information available on the websites of these hospital, and hospitals with online appointment booking features were identified to participate in the study (152 hospitals). Then, the activeness of Internet-based reservation appointment system was evaluated through reserving experimental appointments by researchers after obtaining permission from hospitals via the telephone. Finally, there were only 49 hospitals with active online appointment booking systems that delivered their online services through 31 active appointment booking systems. The selection process of systems that offered online appointment booking services is presented in Figure 1.

Next, the above appointment booking systems were evaluated using a localized checklist. The checklist used in this study was taken from Subramani and Bexci et al.'s study under the title of "Providing a new online appointment model" (1). This model was used to prepare the checklist because of having important features, such as integration of items proposed for evaluation of online medical appointment booking systems. The main structure of the checklist (44 items) in 6 sections, included preappointment booking facilitations (13 items), and demographic characteristics of the patients (22 items), clinical data (3 items), details of the health insurance ( 1 item), post processing facilitation ( 4 items) and internet security features ( 1 item). Finally, the localized checklist of 59 items was designed by adding new items to pre-appointment booking facilitations ( 8 items), patient demographic information ( 5 items), clinical data ( 1 item), and post processing facilitation ( 1 item) for qualitative evaluation of appointment systems in hospital outpatient departments in Iran. The new items were extracted according to preliminary assessment of online appointment systems, websites, national directives, and guidelines in this area. The checklist score was calculated based on existence or nonexistence of all 59 items (yes $=1$ and no $=0$ ). The quality of studied appointment booking systems was classified with respect to each score and total assessment score. Percentages below $50 \%$, between $50 \%$ and $75 \%$, and above $75 \%$, were considered as weak, average, and good, (if $75 \%$ of answers were positive, the booking system had a good condition, between $50 \%$ and $70 \%$ showed moderate, and less than $50 \%$ of an- 


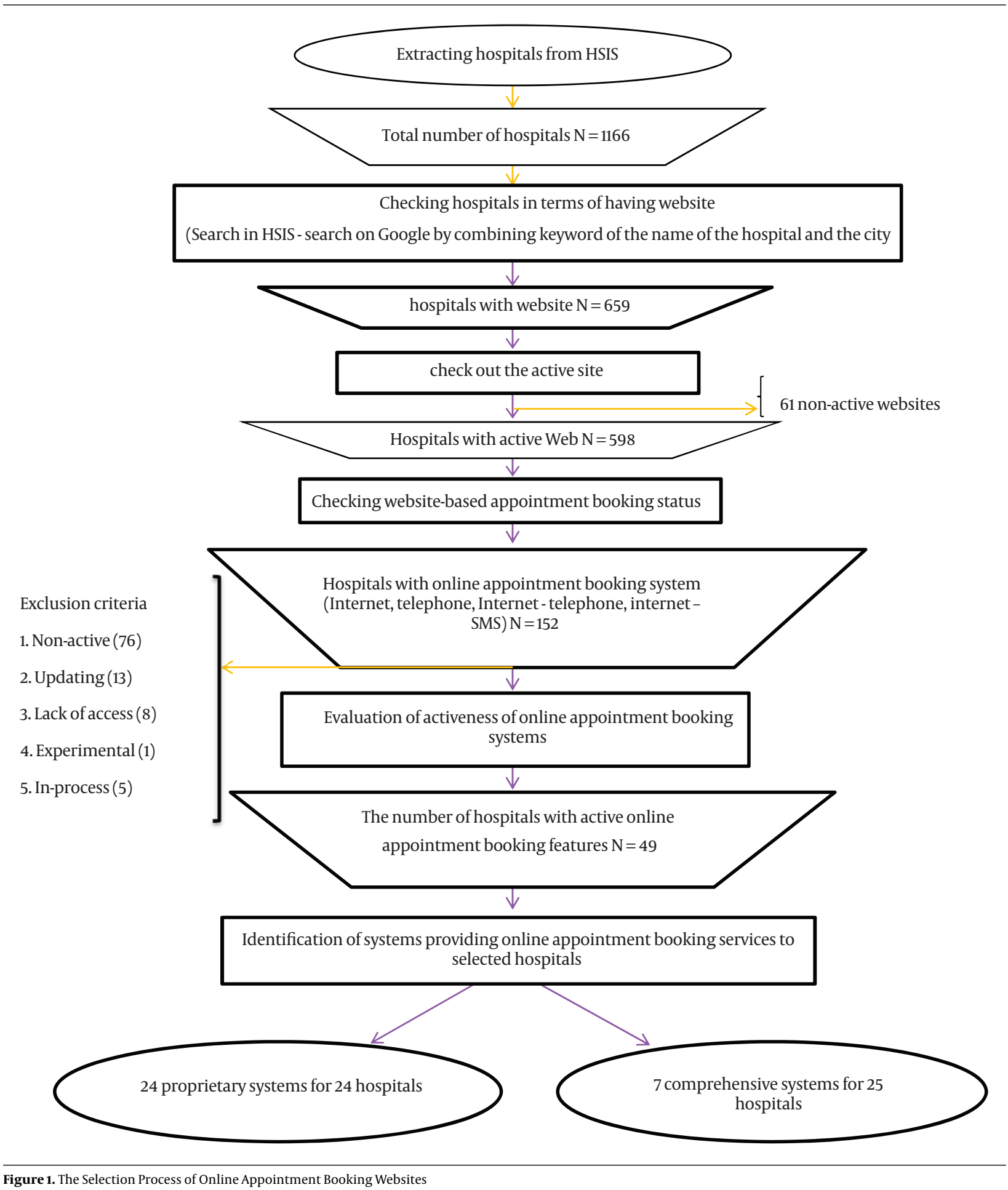

swers indicated a positive condition; the website is not proper enough this scale ranges provided by researchers in this research project).
The checklist content validity was evaluated through survey with relevant experts in the field of health information management, health information technology, com- 
puter engineering, and health services management ( 3 out of every field of study). Also, the inter-rater reliability was evaluated for 25 systems (the online outpatient booking system) that were selected randomly using Cohen's kappa coefficient $(\mathrm{K}=0.82)$. The reliability was determined by 2 researchers separately and then the acquired results by each researcher was compared and the agreement rete of the checklist was calculated.

\section{Results}

The results of the study of characteristics of hospitals in Iran showed that the largest number of hospitals (65.52\%) were owned by the University of Medical Sciences, among which $38.91 \%$ and $27.74 \%$ were active in the treatment and educational-treatment fields, respectively (Table $1)$.

Table 1. Characteristics of Studied Hospitals

\begin{tabular}{|cc|}
\hline Characteristics of Hospitals & Number (\%) \\
\hline Type of activity and dependency & \\
\hline Collegiate-educational-treatment & $323(27.74)$ \\
\hline Collegiate- treatment & $453(38.91)$ \\
\hline Non- collegiate & $387(33.24)$ \\
\hline Property type & \\
\hline University of Medical Sciences & $764(65.52)$ \\
\hline Social security & $67(5.75)$ \\
\hline Armed Forces & $59(5.06)$ \\
\hline Charity & $33(2.83)$ \\
\hline Private & $184(15.78)$ \\
\hline Other & $59(5.06)$ \\
\hline Type of care & 1166 \\
\hline General & $918(78.86)$ \\
\hline One specialty and subspecialty & $114(9.79)$ \\
\hline Number of hospitals & 119949 \\
\hline
\end{tabular}

Out of a total of 1166 Iranian hospitals, 507 lacked a website and internet-based appointment procedure existed only in 49 hospitals. The outpatient appointment booking system in hospitals with an active website is investigated in Table 2 (598 hospitals). The Table shows that the most and least widely used appointment booking methods were in person (75.84\%) and phone-SMS (0.08\%), respectively. Moreover, the phoning method was used more (10.8\%) than the sheer internet-based method (9.43\%).

The frequency of items in the appointment systems is shown in Table 3 based on the studied sections. The most
Table 2. The Appointment-Making Procedure for Outpatients Visiting the Studied Hospitals

\begin{tabular}{|lcc|}
\hline Type of Outpatient Appointments & Number & Percent \\
\hline In-person & 316 & 52.84 \\
\hline Remote & & \\
\hline SMS & 2 & 0.33 \\
\hline Call & 127 & 21.24 \\
\hline Internet & 110 & 18.40 \\
\hline Call-SMS & 1 & 0.17 \\
\hline Call- Internet & 37 & 6.18 \\
\hline All items & 5 & 0.84 \\
\hline Total & 598 & 100 \\
\hline
\end{tabular}

frequent item was patient's name in the demographics section (27 websites). The highest frequencies were related to search by the doctor (25 websites), patients' telephone number (25 websites), and appointment date (19 websites) in the section of pre-appointment booking facilitations. The following items didn't exist in any of the systems: alternative choice of date, alternative choice of time, user email, marital status, occupation, name of employer/company, photo upload, emergency contact person, relationship of the patient to the emergency contact person, personal address of the emergency contact, phone number for emergency contact, passport number and date of expiry of visa for foreign nationals, referee name, blood group and upload medical case.

The results of the study on the online outpatient booking systems in Iranian hospitals are shown in Table 4. All appointment systems were weak (below 50\%) in terms of mean score in each section and overall score. The highest percentage score was obtained in Internet security features (45\%) and details of health insurance (42\%), respectively, while the "clinical data" had the lowest score (7.25\%). The highest and lowest total assessment score for the appointment booking systems were 17 and 2 (out of total 59), respectively. The highest score belonged to the appointment booking system of "Bojnourd Imam Ali hospital, North Khorasan province" with a percentage total score of $28.81 \%$.

\section{Discussion}

The present study was the first study, which comprehensively investigated the outpatient appointment booking system approach and features of online outpatient's appointment booking in all hospitals of Iran (public and private, etc.). Results of the current study showed that 
Table 4. Assessment Scores of Sections Evaluated in the Online Outpatient Appointment Booking Systems in Iran

\begin{tabular}{|c|c|c|c|c|c|}
\hline Sections & $\begin{array}{l}\text { Score and the Number of Items } \\
\text { Per Section }\end{array}$ & Average (\%) Obtained Scores & The Least & The Highest & Standard Deviation \\
\hline $\begin{array}{l}\text { Pre-appointment booking } \\
\text { facilitations }\end{array}$ & 21 & $4.48(21.3)$ & 0 & 9 & 2.11 \\
\hline $\begin{array}{l}\text { Patient demographic } \\
\text { characteristics }\end{array}$ & 27 & $3.68(13.62)$ & 0 & 9 & 1.85 \\
\hline Clinical Data & 4 & $0.29(7.25)$ & 0 & 3 & 0.69 \\
\hline Details of health insurance & 1 & $0.42(42)$ & 0 & 1 & 0.50 \\
\hline Post Processing Facilitation & 5 & $0.77(15.4)$ & 0 & 3 & 0.99 \\
\hline Internet Security features & 1 & $0.45(45)$ & 0 & 1 & 0.50 \\
\hline Total number of websites & 59 & $10.09(17.10)$ & 0 & 17 & - \\
\hline
\end{tabular}

outpatient appointment in Iranian hospitals is done more traditionally and through the patient's in-person visit to medical care centers, and an online appointment booking system is used only in $13.03 \%$ of hospitals. In a study entitled "Evaluation of online appointment booking websites of (Iran) University of Medical Sciences", Bastani et al. reached similar conclusions about the outpatient appointment approach (26). However, studies in this field showed that in-person appointment leads to problems, such as forgetting appointments and increased waiting times, long lines of patients, stressful situation for hospital or clinic staff, and large amount of paper work $(5,6,13,15,16,26)$, therefore, the online appointment reservation system has been proposed as a replacement to deal with these problems $(1,15,27-30)$.

The results of this study revealed that $43.5 \%$ of Iranian hospitals lacked a website and 5\% of them had inactive websites. In previous studies on quality assessment of websites of government (27) and private hospitals (31) in Iran, similar to the current study, a large number of hospitals were lacking websites in the "appointments registration system" in China (28), although the rapid growth of information technology in the field of health has revealed the increasing importance of hospital websites in providing information and services. Also, hospital websites are considered as an appropriate system for the exchange of information between patients and hospital care providers, while attracting more clients (27).

Qualitative evaluation of online appointment booking systems of the studied hospitals showed that these systems had low quality, in terms of percentage of the average score of the studied sections (pre-appointment booking facilitations, patient demographic characteristics, clinical data, details of health insurance, post processing facilitation and Internet security features) and total assessment score (less than 50\%). In a study on hospital websites of Iran University of Medical Sciences, the online appoint- ment booking systems were average and weak in terms of being user-friendly and integrative (26). Also, in a study on the assessment of the quality of government hospitals' websites, the quality of these was reported at a low level (27).

However, the quality of most public hospitals' websites in China was good in terms (activity) of content and design (32). Hence, codification and communication of national standards for designing hospital websites, periodical evaluation of appointment booking websites by Ministry of Health and survey of the users of this method could enhance the qualitative level of these websites.

The findings of this study showed that there was the possibility to search by last name and medical specialty in more online appointment booking studied systems (80.64 $\%$. In one study, Bastani et al. showed that online appointment booking systems in most healthcare centers affiliated to the Universities of Medical Sciences, Iran, could be searched based on the physicians' specialization, physicians' last names, and days of the week (26). Consideration of the ability to search based on the characteristics required by the users could enhance the quality of these systems.

According to the results of the present study, 9.67\% of the appointment-making systems had a post-appointment reminding option. Also in one study by Finkelstein et al., entitled "Priorities of appointment reminder systems and patient", the use of this facility was reported in $2.66 \%$ of all systems studied in Colombia (33), although in a study from Norway, $45.45 \%$ of hospital appointment systems were able to recall the appointment (34), which is not consistent with the current study. The reviewed studies revealed that post-appointment recall probability significantly contributed to the system flexibility, time management(15), reduction in the number of missed appointments $(14,15,24$, $25,33,34)$ and costs $(33,34)$.

One of the important features of the online appoint- 
ment booking systems is the possibility of canceling the appointments $(10,11,14)$, and the results of this study showed that only 3 of the 31 studied systems had this potential. The possibility of canceling the appointment and the appointment replacement are considered as one of the important features in the web-based appointment booking system architecture (6), which reduce missed appointments and improve the efficiency and effectiveness of care $(1,5)$.

The results of this study showed that physicians' weekly schedule is available only in $29.3 \%$ of hospital appointment booking systems. However, in one study, Bastani et al. showed that most online appointment booking systems could provide adequate information in various fields, such as programs for physicians' presence in hospitals, specialties, beds, etc. (26). Dissimilarity of the results could be because the samples under study included online appointment booking systems and in cases where several hospitals used an integrated system of online making appointments, only one appointment booking system was calculated. Making necessary reports to clients has been proposed as one of the important features of webbased appointment-making systems (21) and consideration of this feature in designing online appointment booking systems will lead to the desired results, such as avoiding clients' congestion and wasting time of physicians and patients (22). Offers of appropriate information could encourage more people to use this system and facilitate its application $(6,35,36)$.

Results of the current study also showed that a comprehensive online appointment-making system was used only in 7 University of Medical Sciences in Iran (25 hospitals) and online special appointment booking services were used in 24 hospitals. However, the study of Protti et al. in Andalucia showed that a centralized appointment booking service is used for reservations of more than $25 \%$ of primary care physicians' appointments (29). Using comprehensive online appointment booking systems could be effective in creating benefits, such as being informed about all health care facilities in affiliated centers, full knowledge of physicians' programs $(10,37)$, integrated search feature, appropriate distribution of specialty services, being user friendly (37), leading to patient satisfaction $(9,12,38)$, saving time and facilitating scheduling in clinics. Therefore, the need to design, develop and implement comprehensive online appointment booking systems must be considered as a priority by medical universities. However, there were some problems in this study. One, some systems were inactive during the study. Another problem was that to check some items, it was required to have a test reservation that was assigned one at a time.

\subsection{Recommendations}

Since the online appointment booking system is a win-win solution for patients and physicians, it is recommended to design a national standard for creating websites for appointment booking online appointment by the ministry of health and communicate this to all provinces. Furthermoer, the system should be periodically evaluated based on national standards. Also, designing a comprehensive website for each province must be placed on the agenda to avoid confusion and save time and costs for the visitors.

Furthermore, it is recommended to consider the following features in available appointment-making websites as well as in the design of new websites: possibility to change the appointment time, registration of user's email, personal address for emergency calls, patients' phone numbers for emergency contacts, passport number and date of expiry of visa for foreign nationals, referee number, blood group, and upload medical case. It is also important for University of Medical Sciences and ministry of health to encourage hospitals (covered by medical universities and other hospitals) to use appropriate policies, in order to design and implement remote appointment booking systems and in particular online appointment booking websites and use incentive mechanisms for leading hospitals in this field. Also, patients should be made aware of the advantages of this system and be encouraged to use it.

\subsection{Conclusion}

Increased satisfaction of the clients of healthcare centers is an important pillar of "health transformation scheme", implemented by the ministry of health and Medical Education.

In this regard, all hospitals are required to convert their appointment-making process to an online appointment booking system in order to improve the level of patient satisfaction, and this issue should be included in their validation.

\section{Footnote}

Funding/Support: This study was funded by the student research committee of Lorestan University of Medical Sciences under Grant 1943.

\section{References}

1. Subramani R, Bexci M. Towards effective use of information and communication technology (ICT) application in the healthcare management: a descriptive study of online appointment system services of hospitals in India. Int J Manag Sci Technol. 2013;1(7):1-26. 
2. McMullen MJ, Netland PA. Lead time for appointment and the noshow rate in an ophthalmology clinic. Clin Ophthalmol. 2015;9:513-6. doi: 10.2147/OPTH.S82151. [PubMed: 25834388].

3. Yeon $\mathrm{N}$, Lee $\mathrm{T}$, Jang $\mathrm{H}$. Outpatients appointment scheduling with multi-doctor sharing resources. Simulation Conference (WSC). 2010. p. 3318-29.

4. Cao W, Wan Y, Tu H, Shang F, Liu D, Tan Z, et al. A web-based appointment system to reduce waiting for outpatients: a retrospective study. BMC Health Serv Res. 2011;11:318. doi: 10.1186/1472-6963-11-318. [PubMed 22108389].

5. Nakhaee N, Oroomiei N, Amiresmaili M, Nekoeimoghadam M, Mohammadi B, Mirzaei S. Challenges of appointment fixing in private physician offices from the viewpoint of physicians and secretaries [In Persian].J Health Dev. 2012;1(3):207-20.

6. Idowu AP, Adeosun OO, Williams KO. Dependable online appointment booking system for NHIS outpatient in Nigerian teaching hospitals. Int J Comput Sci Inf Technol. 2014;6(4):59. doi: 10.5121/ijcsit.2014.6405.

7. Pationt Appointment Reservation system Vilnius University Hospital Santariskes clinics Baltic Institute of Information Technologies. 2008. Available from: http://www.biti.lt/get.php?f.93.

8. Adedokun A, Idris O, Odujoko T. Patients' willingness to utilize a SMSbased appointment scheduling system at a family practice unit in a developing country. Prim Health Care Res Dev. 2016;17(2):149-56. doi: 10.1017/S1463423615000213. [PubMed: 25851031].

9. Nazia S. Online appointment scheduling system for hospitals-an analytical study. Int J Innov Res Sci Eng Technol,. 2014.

10. Gowthem SS, Kaliyamurthie KP. Smart appointment reservation system. Int J Innov Res Sci Eng Technol. 2015;4(6):3985-94.

11. Sherly I, Mahalakshmi A, Menaka D, Sujatha R. Online appointment reservation and scheduling for healthcare-A detailed study. Int Innov Res Comput Commun Eng. 2016;4(2):2053-9.

12. Obulor R, Eke B. Outpatient queuing model development for hospital appointment system. Int J Sci Eng Appl Sci. 2016;2(4):15-22.

13. Saremi A, Jula P, ElMekkawy T, Wang GG. Bi-criteria appointment scheduling of patients with heterogeneous service sequences. Expert Syst Appl. 2015;42(8):4029-41. doi:10.1016/j.eswa.2015.01.013.

14. Taylor NF, Bottrell J, Lawler K, Benjamin D. Mobile telephone short message service reminders can reduce nonattendance in physical therapy outpatient clinics: a randomized controlled trial. Arch Phys Med Rehabil.2012;93(1):21-6. doi:10.1016/j.apmr.2011.08.007. [PubMed: 22000821].

15. Pare G, Trudel MC, Forget P. Adoption, use, and impact of e-booking in private medical practices: mixed-methods evaluation of a twoyear showcase project in Canada. JMIR Med Inform. 2014;2(2). e24. doi: 10.2196/medinform.3669. [PubMed: 25600414].

16. Dusheiko M, Gravelle H. The impact of Choose and Book on outpatient appointment non-attendances. Aix en Provence: CES-HESG conference; 2012. Available from: http://www.ces-asso.org/autres-conferences.

17. Green J, McDowall Z, Potts HW. Does Choose and Book fail to deliver the expected choice to patients? A survey of patients' experience of outpatient appointment booking. BMC Med Inform Decis Mak 2008;8:36. doi: 10.1186/1472-6947-8-36. [PubMed: 18673533].

18. Chen SC, Jong D, Lai MT. Assessing the relationship between technology readiness and continuance intention in an E-appointment system: relationship quality as a mediator. J Med Syst. 2014;38(9):76. doi: 10.1007/s10916-014-0076-3. [PubMed: 25007955].

19. Chen SC, Liu SC, Li SH, Yen DC. Understanding the mediating effects of relationship quality on technology acceptance: an empirical study of e-appointment system. J Med Syst. 2013;37(6):9981. doi: 10.1007/s10916013-9981-0. [PubMed: 24141491].

20. Ellingsen G, Obstfelder A. Collective expectations-individual action implementing electronic booking systems in Norwegian health care. Int J Med Inform. 2007;76 Suppl 1:S104-12. doi 10.1016/j.ijmedinf.2006.05.029. [PubMed: 16782398].
21. Darvish N, Vaezi M. Modeling and scheduling intelligent method's application in increasing hospitals'efficiency. J Theor Appl Inf Technol. 2011;24(2).

22. Zhang X, Yu P, Yan J, Ton AI. Using diffusion of innovation theory to understand the factors impacting patient acceptance and use of consumer e-health innovations: a case study in a primary care clinic. BMC Health Serv Res. 2015;15:71. doi: 10.1186/s12913-015-0726-2. [PubMed: 25885110].

23. Sims H, Sanghara H, Hayes D, Wandiembe S, Finch M, Jakobsen H, et al. Text message reminders of appointments: a pilot intervention at four community mental health clinics in London. Psychiatr Serv. 2012;63(2):161-8. doi: 10.1176/appi.ps.201100211. [PubMed: 22302334].

24. Zhang M, Zhang C, Sun Q, Cai Q, Yang H, Zhang Y. Questionnaire survey about use of an online appointment booking system in one large tertiary public hospital outpatient service center in China. BMC Med Inform Decis Mak. 2014;14:49. doi: 10.1186/1472-6947-14-49. [PubMed: 24912568].

25. Nuti L, Turkcan A, Lawley MA, Zhang L, Sands L, McComb S. The impact of interventions on appointment and clinical outcomes for individuals with diabetes: a systematic review. BMC Health Serv Res. 2015;15:355. doi: 10.1186/s12913-015-0938-5. [PubMed: 26330299].

26. Bastani P, Garavand A, Abhari S. Evaluating the e-booking system through Iranian Medical University centers' Websites [In Persian].J Health Biomed Inf. 2016;2(4):219-28.

27. Salarvand S, Samadbeik M, Tarrahi MJ, Salarvand H. Quality of Public Hospitals Websites: A Cross-Sectional Analytical Study in Iran. Acta Inform Med. 2016;24(2):130-3. doi: 10.5455/aim.2016.24.130-133. [PubMed: 27147806].

28. Yu W, Yu X, Hu H, Duan G, Liu Z, Wang Y. Use of hospital appointment registration systems in China: a survey study. Glob J Health Sci. 2013;5(5):193-201. doi:10.5539/gjhs.v5n5p193. [PubMed: 23985121].

29. Protti D, Johansen I, Perez-Torres F. Comparing the application of Health Information Technology in primary care in Denmark and Andalucia, Spain. Int J Med Inform. 2009;78(4):270-83. doi: 10.1016/j.ijmedinf.2008.08.002. [PubMed: 18819836].

30. Gauld R, Derrett S. Solving the surgical waiting list problem? New Zealand's 'booking system'. Int J Health Plann Manage. 2000;15(4):25972. doi: 10.1002/hpm.596. [PubMed: 11246897].

31. Alipour-Hafezi M, Karimi M, Tofighi S. Conceptual Model to Improve Portal Services of Private Hospitals in Tehran [In Persian].J Health Adm. 2015;17(58):42-57.

32. Joaquin Mira J, Llinas G, Tomas O, Perez-Jover V. Quality of websites in Spanish public hospitals. Med Inform Internet Med. 2006;31(1):23-44. doi: 10.1080/14639230500519940. [PubMed:16754365].

33. Finkelstein SR, Liu N, Jani B, Rosenthal D, Poghosyan L. Appointment reminder systems and patient preferences: Patient technology usage and familiarity with other service providers as predictive variables. Health Informatics J. 2013;19(2):79-90. doi: 10.1177/1460458212458429. [PubMed: 23715208].

34. Hasvold PE, Wootton R. Use of telephone and SMS reminders to improve attendance at hospital appointments: a systematic review. J Telemed Telecare. 2011;17(7):358-64. doi: 10.1258/jtt.2011.110707. [PubMed: 21933898].

35. Mardaninezhad A, Khanjani T, Farhadi I, Amirimoghadam M. Evaluate websites of virtual branch Medical Universities of Iran and the world [In Persian]. Scientific Information Database. 2012;(2):179-90.

36. Stevenson JS. Appointment systems in general practice. How patients use them. Br Med J.1967;2(5555):827-9. [PubMed: 6029148].

37. Aladwani AM. An empirical test of the link between web site quality and forward enterprise integration with web consumers. Bus Process Manag J. 2006;12(2):178-90. doi: 10.1108/14637150610657521.

38. Aslani N, Zhang J. Integration of simulation and DEA to determine the most efficient patient appointment scheduling model for a specific healthcare setting. J Ind Eng Manag. 2014;7(4):785-815. doi: 10.3926/jiem.1058. 
Table 3. Frequency of Items Available on the Studied Websites ${ }^{\mathrm{a}}$

Items in Each Section

The Number of Websites (\%)

Pre-appointment booking facilitations

1- Number of hospital records

$2(6.45)$

2-Search by doctor

$25(80.64)$

3- Search by city

$1(3.22)$

4-Search by hospital

$10(32.25)$

5- Search by specialty

$18(58.06)$

6-Appointment category (first time or follow-up)

$8(25.80)$

7- Type of appointment (consultation / diagnosis / and check-up)

$2(6.45)$

8- Physicians' profile

1 (3.22)

9- Physicians' apps

9 (29.03)

10-Appointment date

19 (61.29)

11- Choice of alternative day

$0(0)$

12-Appointment time

18 (58.06)

13- Choice of alternative time

$0(0)$

14-Search by treatment

1(3.22)

15-Specialty category (including general options)

$1(3.22)$

16-Specialty name (eye surgeon and specialist ,corneal surgery and ...)

$4(12.90)$

17- Date and time of reservation time

$6(19.35)$

18- Services

$4(12.90)$

19-Type of service

2 (6.45)

20- Code admission

4 (12.90)

21- Proof of admission

4 (12.90)

\section{Clinical Data}

1- Blood group

2-Upload medical case

$0(0)$

3- Patient message / remarks / details about symptoms

3 (9.67)

4-Shift

$3(9.67)$

Post processing facilitation) (authentication/verification/cancel)

1- Immediate SMS / phone / Email confirmation of using online appointment booking

2- Approval number of appointments

$12(38.70)$

3- Printing options

$4(12.90)$

4- Cancellation option

3 (9.67)

\section{Patient demographic characteristics}

1-User's email

2- User name

2 (6.45)

3-User's mobile or telephone number

3 (9.67)

4- Patients' Email

$6(19.35)$

5-Patient name

27 (87.09)

6- Patient telephone / mobile

$25(80.64)$ 


\begin{tabular}{|c|c|}
\hline 7-Date of birth & $4(12.90)$ \\
\hline 8- Nationality & $2(6.45)$ \\
\hline 9-Gender & $8(25.80)$ \\
\hline 10- Name of father / spouse & $14(45.16)$ \\
\hline 11- Marital status & $0(0)$ \\
\hline 12- Patient address & $1(3.22)$ \\
\hline 13- Job & $0(0)$ \\
\hline 14- Name of employer / company & $0(0)$ \\
\hline 15- Religion & $1(3.22)$ \\
\hline 16- Photo uploading & $0(0)$ \\
\hline 17- Person name for emergency contact & $0(0)$ \\
\hline 18- Relationship of the patient to the emergency contact person & $0(0)$ \\
\hline 19-Address of introduced individual for emergency contact & $0(0)$ \\
\hline 20- Phone number of the introduced individual for emergency contact & $0(0)$ \\
\hline 21- Passport no and date of expiry of visa & $0(0)$ \\
\hline 22-Referee number & $0(0)$ \\
\hline 23- National ID No & $14(45.16)$ \\
\hline 24- Age & $3(9.67)$ \\
\hline 25- Birth certificate No & $1(3.22)$ \\
\hline 26-Appointment number & $2(6.45)$ \\
\hline 27- Physician address & $1(3.22)$ \\
\hline \multicolumn{2}{|l|}{ Internet security features } \\
\hline 1-Security code & $14(45.16)$ \\
\hline \multicolumn{2}{|l|}{ Details of health insurance } \\
\hline 1-Insurance companies and health insurance number & $13(41.93)$ \\
\hline
\end{tabular}

${ }^{\mathrm{a}}$ The total number of websites $=31$. 